\title{
INTERVENCIÓN SOCIAL: REPRESENTACIONES SOCIALES Y PRÁCTICAS DE ESTUDIANTES DE-TRABAJO SOCIAL
}

\author{
Liliana Pérez Mendoza² \\ Yudis del Carmen Díaz Flórez ${ }^{3}$ \\ Graciela Isabel Páez Rodríguez ${ }^{4}$
}

\section{Resumen}

El artículo presenta los resultados del estudio referido a las representaciones sociales y prácticas de intervención social de estudiantes del programa de Trabajo Social de la Universidad de Cartagena (Colombia), las cuales fueron identificadas a través del análisis del discurso, expresado en entrevistas semiestructuradas dirigidas a estos. Los principales hallazgos dan cuenta de una reproducción lingüística acrítica de los discursos que sobre estos temas manejan los docentes en el aula o en actividades formativas extraacadémicas como semilleros y proyectos de investigación, evidenciándose representaciones sociales y prácticas de este tipo descontextualizadas de las problemáticas locales. [Descriptores: representaciones sociales, prácticas, discursos, intervención social].

1 El artículo presenta resultados de la investigación "Discursos, representaciones y prácticas sobre intervención social y ciudadanía de estudiantes del programa de Trabajo Social de la Universidad de Cartagena, Año 2010", que se enmarca en el proyecto "Intervención social, ciudadanía y contexto en las unidades académicas de Trabajo Social de la costa caribe colombiana" dirigida por los docentes de la Universidad de Cartagena Liliana Pérez Mendoza, Nancy Bolaño Navarro y Kenia Victoria Cogollo.

2 Docente, investigadora de la Facultad de Ciencias Sociales y Educación de la Universidad de Cartagena, Colombia.

${ }_{3}$ Trabajadora social e investigadora Colciencias, Universidad de Cartagena, Colombia.

4 Trabajadora social y auxiliar investigación, Universidad de Cartagena, Colombia. 


\section{Abstract}

The article presents results of the study referred to social representations and practices of social intervention of students of Social Work at the University of Cartagena (Colombia), which were identified through discourse analysis, expressed in semistructured interviews aimed at these. The main findings realize an uncritical reproduction of the discourses that handle teachers about these topics in the classroom or in extracurricular activities, such as seed training and research projects, evidencing social representations and practices of such decontextualized local issues. [Keywords: social representations, practices, discourses, social intervention].

\section{Introducción}

Cartagena de Indias es una de las ciudades colombianas con más avances en materia de turismo, industria y comercio, así como un amplio crecimiento poblacional y expansión territorial, lo cual ha incidido directa o indirectamente en la generación y arraigo de problemáticas sociales. Según datos de la Secretaría de Planeación Distrital (2009), esto se evidencia en un índice del 29.5 por ciento de población en situación de pobreza, correspondiente a 258.581 personas; una población en situación de pobreza extrema que asciende a 256.335 personas, lo que equivale al 29.2 por ciento de esta, y un número aproximado de 26.645 viviendas construidas en zonas de alto riesgo no mitigable (Corvivienda, 2007). Esto indica que alrededor del 58 por ciento de los cartageneros se encuentran en situación de vulnerabilidad geológica. Además, una tasa de desempleo creciente del 13.5 por ciento y de inseguridad ciudadana del 65.4 por ciento, manifestada en homicidios por sicariatos, hurtos, riñas (Cartagena Como Vamos, 2010).

Ante esta realidad, las intervenciones sociales cobran vital importancia hacia el impulso de procesos sociales, formativos, de promoción y acompañamiento, que potencien ciudadanos autogestores, participantes, autónomos y corresponsables con el desarrollo y la justicia social en su contexto local. De igual forma, es importante que desde las diversas instituciones, organizaciones y en especial la academia, se concerten e impulsen reflexiones, estudios y estrategias sociales con nuevos argumentos conceptuales y encuadres metodológicos basados en el contexto, y dirigidos hacia la preservación e impulso del mejoramiento de la calidad 
de vida de la población. En tal sentido, en la intervención social hoy se elevan propuestas que señalan el compromiso de esta con el reconocimiento de las personas, como sujetos sociales y ciudadanos, con derechos y deberes, apostándole a la construcción de ciudadanía desde el respeto por las diferencias, la heterogeneidad social, los diferentes discursos y puntos de vista, el fomento de la democracia no sólo como derechos individuales, sino también enfocada desde contextos, que como los educativos propicien relaciones sociales que involucren a otros sujetos (Acevedo, 2003).

De acuerdo con esto, es importante partir por investigaciones que permitan identificar las representaciones sociales y prácticas que sobre intervención social expresan los diferentes actores. En tal indagación, ha de tenerse en cuenta las voces de los sujetos que realizan este tipo de acciones sociales como son los estudiantes de Trabajo Social en este caso, las cuales deberán ser contrastadas posteriormente con la de los docentes y demás actores articulados a su formación profesional. Por lo anterior, resulta acertado el señalamiento de Reguillo (2004) acerca de que "toda acción es susceptible de representación y las representaciones únicamente son aprehensibles a través del discurso, en su sentido no restringido a los códigos verbales" (p. 4). Desde tal perspectiva, en el 2010 se desarrolló la investigación Discursos, representaciones y prácticas sobre intervención social y ciudadanía de estudiantes del programa de Trabajo Social de la Universidad de Cartagena, enmarcado en el proyecto: Intervención Social, Ciudadanía y Contexto en las Unidades Académicas de Trabajo Social de la Costa Caribe Colombiana, dirigido por las docentes Liliana Pérez Mendoza, Nancy Bolaño Navarro y Kenia Victoria Cogollo, del grupo de investigación Cultura, Ciudadanía y Poder en Contextos Locales de la Facultad de Ciencias Sociales y Educación, Universidad de Cartagena.

En este artículo se presentan aspectos relevantes de la investigación, llevada a cabo a través del método investigativo análisis del discurso. Se muestran los significados y prácticas sobre intervención social, desde lo que expresan los estudiantes de último año del programa de Trabajo Social de la Universidad de Cartagena. 


\section{Marco teórico-conceptual}

En el inicio de la investigación se realizó un análisis de las definiciones de diferentes autores sobre intervención social y a partir de allí se asumió una conceptualización de la misma, desde la cual se interpretó las representaciones sociales, prácticas y los discursos de los estudiantes de Trabajo Social sobre esta. La intervención social es definida por Carballeda (2002):

Supone la necesidad de una búsqueda, de una construcción, de una modalidad discursiva diferente, determinada ahora por el sujeto, por su propia palabra, por su singularidad, a la vez que recupera la importancia de los vínculos de ese sujeto con otros, buscando desde allí una resemiotización de aquello que se construyó discursivamente como hegemónico. (p. 111)

Así, se entiende la intervención social como una relación dia-lógica e intersubjetiva entre sujetos intervenidos e intervinientes, donde los discursos o acción lingüística de ambos actores son determinantes en las concepciones, percepciones y orienta-ciones de la misma.

Por eso, resulta vital que en la intervención social del Trabajo Social en este caso, se empiece por identificar y reflexionar acerca de los discursos de todos sus actores frente a la misma, a fin de reconocerlos, diferenciarlos y articularlos en un diálogo sensible y esclarecedor, dirigido a proponer formas emergentes y contextualizadas para entender y desarrollar tal intervención. En este sentido, es importante partir de procesos reflexivos y críticos por parte de los profesionales que realizan esta intervención; que permita explorar y considerar expectativas, sentidos, representaciones y prácticas sociales desde sus voces, lo cual debe ser replicado con el resto de los actores participantes en la misma, a fin de que a propósito de éste, se propicie un diálogo fecundo dirigido a consensuar formas de acción social sensibles al contexto y a sus actores.

Para llegar a lo anterior, resultó clave definir de entrada cómo entender también en la investigación, las representaciones sociales, prácticas sociales y discursos. De acuerdo con Botero (2008), las representaciones sociales hacen referencia:

[...] al mundo de la vida cotidiana, a aquello que no es especializado sino que desde las 
motivaciones, intereses y necesidades colma de sentido las actividades del sujeto en relación. Los objetos son poseedores reales del significado que les confiere la intersubjetividad, cobran vida para convivir entre los sujetos y pueden dar cuenta del sentido. (p. 17)

Es decir, se trata de las significaciones que colectivamente se asumen frente a diferentes hechos, situaciones y objetos, ya sea de manera consensuada o no.

Con respecto a los discursos, estos como acción social, Van Dijk (2001) plantea que "ocurren en un marco de comprensión, comunicación e interacción que a su vez son partes de estructuras y procesos socio-culturales más amplios" (p. 3). Añade Van Dijk (2001), que obedecen a un sentido semiótico que incluye además expresiones no verbales tales como dibujos, cuadros, gestos, acciones, textos, etc. De allí que el discurso se convierta en un evento comunicativo complejo, que según Londoño (2007):

Involucra a actores sociales, esencialmente en los roles de hablante/escribiente y oyente/ lector (pero también en otros roles, como observador o escucha), que intervienen en un acto comunicativo, en una situación específica (tiempo, lugar, circunstancias) y determinado por características del contexto. (p. 3)

En este orden de ideas, podría afirmarse que el sentido de un discurso no se define únicamente desde sí mismo, sino también en el marco del contexto en el que se produce, lo que determina una función básicamente social.

En consecuencia, el discurso debe ser explicado, comprendido y analizado desde el contexto en el que se enuncia y especialmente desde el rol sociocultural, institucional y discursivo del hablante, por cuanto este da cuenta de un conjunto de características importantes alrededor de la comunicación tales como la cultura de los participantes, sus experiencias, estereotipos, prejuicios, actitudes, expectativas, necesidades y demandas. En este caso, lo destacable es la importancia de establecer y considerar el marco contextual particular en el que se originan los discursos de los actores, para su interpretación y comprensión.

Cabe destacar que, en cada contexto se desarrollan procesos históricos, sociales, culturales, económicos y políticos 
que se interrelacionan directa e indirectamente, los cuales van configurando un sistema de representaciones y prácticas sociales expresadas mediante los discursos de sus actores, que develan sus percepciones y usos sociales. Por otro lado, al hablar específicamente de prácticas sociales, desde la pers-pectiva kantiana, Carballeda (2007) afirma que estas hacen referencia a:

Una configuración concreta, no se denomina práctica a cualquier ocupación sino a la efectuación de un fin pensado como consecuencia de ciertos principios metódicos representados en general [...] la práctica interpela al conocimiento desde lo empírico, la pregunta interroga desde el hacer cotidiano, pero requiere de una instancia reflexiva en diálogo con la teoría. (p. 15)

De acuerdo con esta perspectiva, se resalta la práctica social como la realización de un fin, desde un esquema metodológico y empírico que interpela los marcos teóricoreferenciales en que pueden surgir. Tales prácticas se establecen dentro de una dinámica de transformación, en la que surgen y cambian con el devenir histórico de la vida social de los sujetos. Para Castañeda (2004), "toda práctica social puede reinterpretarse en términos de estructuras conversacionales y examinarse como un marco para la acción definido por un conjunto de distinciones lingüísticas" (p. 1). Visto de esta forma, las prácticas y discursos tienen una relación dialéctica que contribuye a su definición y redefinición.

Para el caso de la formación profesional en Trabajo Social, este punto de partida implicará que los estudiantes antes de iniciar sus prácticas académicas realicen un ejercicio guiado de reconocimiento, reflexión y validación de sus concepciones acerca de la intervención social, de cara a validar enfoques, teorías y metodologías aprendidas, pero sobre todo, para establecer las construcciones simbólicas que tienen acerca de la misma, basados en el contexto local, su trayectoria de vida, proyecciones profesionales y reconocimiento de la otredad. Es a partir de estas conceptualizaciones sobre intervención social, representaciones sociales, discursos y prácticas sociales desde donde se interpretan los resultados de la investigación realizada.

\section{Metodología de investigación}

La investigación estuvo orientada desde el método cualitativo análisis del discurso (AD), el cual permite "descubrir 
los significados de las cosas, interpretar lo mejor posible las palabras, los escritos, los textos, los gestos, y en general el comportamiento humano [...] conservando la singularidad del contexto que hace parte" (Dilthey, según citado en Martínez, 2006 , p. 3). Este método ofreció herramientas y técnicas para recoger, procesar e interpretar las representaciones sociales y prácticas, a partir de las voces de los estudiantes de Trabajo Social de la Universidad de Cartagena.

Van Dijk, plantea que al realizar un análisis discursivo es necesario tener en cuenta que "el discurso como acción social ocurre en un marco de comprensión, comunicación e interacción que a su vez son partes de estructuras y procesos socio-culturales más amplios" (según citado por Silva, 2002, p. 4). En ese sentido, se asume que los discursos expresados por los estudiantes en el contexto universitario hacen eco de los discursos de un contexto sociocultural e histórico más amplio.

El proceso metodológico seguido, se realizó a través de tres (3) fases, la primera correspondió a la recolección de información secundaria y primaria. Para la información secundaria se recopilaron textos, documentos y artículos referentes a la categoría de intervención social, procediendo luego a la elaboración de fichas bibliográficas y matrices temáticas, según subcategorías. La información primaria se recolectó mediante entrevistas semiestructuradas dirigidas a los estudiantes de último año vinculados al Programa de Trabajo Social de la Universidad de Cartagena (Colombia). Participaron diez (10) estudiantes, de sexo masculino o femenino (5 por cada uno de los dos últimos semestres).

La segunda fase, se procedió a la organización y análisis de la información, clasificándola de acuerdo a las categorías de análisis propuestas en la investigación, utilizando como apoyo la herramienta para procesamiento de datos cualitativos Atlas-ti. Posteriormente en la fase final se sistematizaron los hallazgos resultantes de la investigación, y se plantearon algunos lineamientos curriculares para la formación profesional en Trabajo Social.

\section{Hallazgos referidos a las representaciones sociales, prácticas y discursos de intervención social}

Dada la riqueza de la información resultante, a continuación se presenta una síntesis de los principales hallazgos. En la indagación realizada en la Universidad de 
Cartagena con los estudiantes de Trabajo Social, los resultados presentados en la matriz $\mathrm{N}^{\circ} 1$ (Ver Apéndice) arrojan que las representaciones sociales que se develan en sus discursos en relación a la intervención social, coinciden con una concepción en la que esta es asumida como un conjunto de acciones sociales, las cuales deben ser organizadas de forma dinámica desde disciplinas como Trabajo Social, apuntando hacia el desarrollo social, el bienestar de los sujetos y el abordaje de problemáticas en contextos y situaciones específicas, que posibiliten la transformación de la realidad social.

Intervención es el conjunto de acciones que como trabajadores sociales llevamos a cabo con fines determinados que apuntan al desarrollo, al bienestar de las personas y que es acorde a las demandas de la sociedad [E3]. ${ }^{5}$

Son aquellas acciones organizadas, son acciones flexibles que te permiten realizar un cambio y hacer partícipe a la comunidad de su propio cambio [E1].

Son esas acciones encaminadas a transformar una realidad presente por una deseada [E8].

Del mismo modo, se entiende la intervención social necesariamente con un carácter teórico y metodológico como fundamento científico para el Trabajo Social, resaltando la importancia de la investigación científica como parte de esta intervención.

Es una acción que implica lo investigativo, lo científico, porque de esta manera se procura la trasformación social [...] [E5].

Es la forma en que, teórico ó metodológica el trabajador social o cualquier otro teórico de las ciencias sociales, llega al campo en el cual decida trabajar una problemática [E2].

Se encontró además que las categorías que los/as estudiantes asocian a la intervención social son: participación, transformación, ética profesional, desarrollo, sostenibilidad, comunidad, cultura, mediación, política, democracia, investigación, práctica, diálogo, responsabilidad social y educación, las

$5 \quad$ E: abreviatura de entrevista las cuales se le asignó un número a cada participante para preservar la identidad de estudiantes. 
cuales están directamente relacionadas con los métodos de intervención social utilizados en el Trabajo Social, tales como caso, grupo y comunidad. (Ver matriz $\mathrm{N}^{\circ} 1$ en Apéndice)

Cuando el actor habla de ética en el trabajo hace referencia a la ética profesional que como trabajador o trabajadora social se debe asumir [E1].

Una categoría para relacionar puede ser la transformación en tanto se parte de una situación inicial y a partir de lo que identificamos en ella [E4].

Considero categorías como lo político, lo ético, lo investigativo, la acción, la transformación, la práctica [E5].

También se evidenció que la fundamentación teóricometodológica de la intervención social identificada en los discursos de los estudiantes está sujeta a los paradigmas de las ciencias sociales y a los métodos de investigación social, aún cuando no existe una identificación clara de sus autores. Tal respuesta es expresión de los conocimientos que los estudiantes han recibido en el aula de clase en diversas asignaturas sobre esta temática.

Constructivismo, paradigma del Interaccionismo simbólico, hermenéutica, fenomenología, bueno en lo que tiene que ver con la investigación [E2]. Los paradigmas hermenéutico, etnográficos, interpretativos, de igual manera la teoría fundamentada, la investigación-acción-participativa, diagnóstico participativo [E5].

Paradigmas, comolaetnografía, lahermenéutica, Interaccionismo simbólico [E10].

Por otra parte, según los resultados de la investigación, estudiantes de Trabajo Social participantes reconocen como discursos contemporáneos de intervención social, en primer lugar, la importancia del tema ético, visto con un sentido humanizador que hace referencia al respeto a la diferencia, a la lucha por el reconocimiento y la garantía de los derechos de las poblaciones vulnerables. (Ver matriz $\mathrm{N}^{\circ} 2$ en Apéndice).

Hoy día el Trabajo Social debe ser un Trabajo Social innovador basado en tendencias contemporáneas que aluden a una ética 
humanizadora, una ética que hace alusión al respeto por la diferencia, que lucha por el reconocimiento de las poblaciones vulneradas en sus derechos, además nos hace un llamado a cambios radicales [E8].

Desde las nuevas tendencias contemporáneas de Trabajo Social se le apunta a una ética que debe estar presente en la intervención social desde cualquier abordaje que se haga en Trabajo Social, se le está apuntando a tener en cuenta a los sujetos sociales como sujetos de derecho, que obtienen sus saberes, se debe trabajar desde las mismas lógicas internas de ellos [E2].

Es válido señalar además que, en tales discursos reconocidos por los/as estudiantes, el tema ético está presente como una constante de la intervención social contemporánea, lo cual se relaciona con lo que Pérez (2008) afirma acerca de que la misma está llamada hoy más que nunca a ser planteada como su centro:

Razones éticas, que recuerden que la justicia social y el bienestar común, son principios que nunca deben negociarse, ni mucho menos, suprimirse, porque es a través de ellos como se puede contribuir a la reconstrucción del proyecto moderno, potenciando autonomía, solidaridad e igualdad entre las personas. (p.3)

Estos discursos podrían asociarse a los planteamientos de Barranco (2004), que la intervención en Trabajo Social es entendida como una acción organizada y desarrollada con las personas, grupos y comunidades, y se orienta hacia el desarrollo humano y el mejoramiento de la calidad de vida de los actores siendo además "una intervención integrada por lo ético, lo epistemológico y metodológico, desde un enfoque global, plural y de calidad" (p.79). Esto indica una intervención social que se asume desde sus propios alcances y generalidades, así como desde necesidades puntuales de problemas abordables por la misma.

Los estudiantes destacan, además, como discursos contemporáneos en la intervención social aquellos que se orientan a la necesidad de implementar la autogestión, como herramienta para la formulación de proyectos, desde 
la participación activa de las comunidades; así como otros orientados a la responsabilidad social de las empresas, el reconocimiento de sujetos de derechos, la integralidad e interdisciplinariedad.

Actualmente se maneja los discursos de las mismas comunidades, los mismos beneficiarios de los proyectos, participen directamente en la construcción de estos proyectos que no seamos nosotros como trabajadores sociales, quienes diseñemos desde un escritorio el proyecto, sino que sea desde la misma comunidad que se dé la creación de estos proyectos desde la participación activa de todos [E9].

Todas estas dinámicas como la globalización, todos estos procesos que actualmente enfrentamos te plantean la necesidad que realices intervenciones de otra manera que redefinas la mirada de estas intervenciones; y por ejemplo te plantean la necesidad de hacer intervenciones interdisciplinarias e integrales [E3].

En tal sentido como se indica en la matriz $\mathrm{N}^{\circ} 2$ (ver Apéndice), los estudiantes afirman impulsar discursos orientados a la superación de la pobreza, la equidad de género, el reconocimiento de los derechos humanos y la diversidad cultural, retomando la necesidad de la autogestión, esta vez como herramienta para el desarrollo de los procesos sociales desde la educación y la participación, a partir de una conciencia crítica frente a la realidad social.

El contexto nos está demandando nuevas formas de intervenir, nuevas miradas, nos está exigiendo incluso que tomemos posturas frente a todas estas situaciones que enfrentamos día a día en el tema de género por ejemplo tenemos grandes avances y creo que nos hemos ido incursionando por allí, en el tema de familias seguimos posicionándonos y creo que la misma dinámica nos muestra que tenemos bastante por hacer [E5].

Considero que a partir de que tengamos en cuenta lo importante que es entender al otro como diferente, asimismo vamos a respetarlos 
y hacerles un reconocimiento como sujetos también de derechos y reconocernos a nosotros mismos, además por otro lado creo mucho en los discursos de autogestión, autonomía, de los sujetos sociales [E2].

Discursos como la superación de la pobreza, la equidad de género, el restablecimiento de derechos, y también el papel que enfrenta el trabajador social, frente a las problemáticas sociales [E7].

Por ello, en relación con las prácticas contemporáneas de intervención social que reconocen los estudiantes, están aquellas relacionadas con los temas de equidad de género, derechos humanos, familia, política, responsabilidad social y desarrollo comunitario. Prácticas que fundamentadas desde lo teórico y gestadas desde los actores apuntan a la producción de conocimiento, tal como se evidencia en la matriz $\mathrm{N}^{\circ} 3$ (ver Apéndice).

Hoy está visto las prácticas en torno al género, derechos humanos, la familia, lo político [E8].

Un tipo de prácticas fundamentada y que busca más que la dependencia de las personas a los procesos que desarrollamos la autogestión de éstas, que éstas personas sean autogestoras, se empoderen, se formen porque en la medida que tú te formes conoces y en la medida que conoces exiges [E3].

Del mismo modo, los estudiantes afirman impulsar prácticas en su intervención social orientadas hacia la diversidad cultural y la formación de líderes en poblaciones vulnerables, así como, a la equidad de género, al reconocimiento y respeto de los sujetos y al fortalecimiento de la autogestión como instrumento para el empoderamiento de los derechos por parte de los sujetos sociales. De manera particular, los estudiantes destacan en su ejercicio profesional el desarrollo social desde la potencialización de los sujetos y la intervención integral con familias.

Desde mi práctica impulso el desarrollo local, impulso el desarrollo social, el mejoramiento de la calidad de vida desde adentro, la potencialización del ser humano, mirar lo que en realidad quiere la población con las cuales se está interviniendo [E6]. 
Aquellas acciones que van encaminadas a la formación de líderes [E2].

Dentro de las prácticas como profesional el "respeto al otro", dentro del respeto enmarca el reconocer a la otra persona, y no reconocerlo desde esa es la persona con la que estoy trabajando, esa es la persona que me puede brindar a mí este tipo de información, sino reconocerlo en la medida que le dé significado a lo que esa persona me está diciendo y trasmitiendo durante todo el proceso [E4].

Considerando la respuesta de los estudiantes, acerca de la equidad de género como una de sus prácticas en la intervención social, pareciera entenderse que este enfoque sólo hace referencia exclusivamente al caso de las reivindicaciones femeninas, de sus derechos y participación social, entendiéndose el género referido solamente a la defensa de las mujeres.
Aun cuando hoy se siguen reproduciendo prácticas asistenciales y de servicios yo creo que la profesión hoy se está encaminando a trabajar por la equidad de género, por hacer que el papel de la mujer sea reconocido y menos vulnerado dentro de la sociedad, además de temas como la ciudadanía y la interculturalidad [E2].

Por otrolado, pese a que desde los discursos expresados por los/as estudiantes se adviertan elementos teóricosmetodológicos en relación a sus prácticas y representaciones sociales sobre intervención social, también en sus respuestas, pareciera advertirse una reproducción lingüística de discursos aprehendidos en el aula, que muestran débiles despliegues propios, haciéndose necesario en la formación profesional en Trabajo Social, el potenciamiento, reflexión, criticidad, debate y construcción de concepciones con relación a la intervención social y su contextualización, que trasciendan la reproducción de discursos e instrumentalización de las prácticas y se articulen a una visión más integral de la realidad abordada. Y es que la intervención social, de acuerdo a Tejos (2005) ha de suponer tensión en:

El desarrollo de un esfuerzo simultáneo por
generar capacidades en las personas, familias 
y comunidades, de manera tal, que a través del despliegue de sus capacidades y utilización de sus potencialidades, pueden dichas personas, familias y comunidades, iniciar un proceso gradual y sustentable de mejoramiento de su calidad de vida. (p.6)

Se trata desde esta perspectiva de apuntar hacia un desarrollo social, endógeno y sostenible, que en el caso de los profesionales en Trabajo Social será posible, si desde la formación universitaria se empieza a potenciar tal fin, y a asumir la intervención social como una relación de doble vía entre interventores e intervenidos que, enfaticen en la puesta en común y acuerdos acerca de las representaciones sociales, prácticas y discursos que sobre la misma éstos actores tienen.

\section{Conclusiones}

De acuerdo con lo anterior, puede concluirse en primera instancia que, las representaciones sociales que los estudiantes de último año de Trabajo Social tienen de intervención social, se limitan a un accionar que si bien está permeado por elementos teóricos, metodológicos e investigativos como lo plantean en sus discursos, estos parecieran ser el eco de la información que han recibido en el aula. Muestra de ello, es el hecho de que, si bien en los discursos de los estudiantes se aprecia la identificación de algunos autores desde Trabajo Social y las ciencias sociales, no existe una identificación clara de cuáles de estos le aportan a la intervención social específicamente y no se evidencia una apropiación y diferenciación de los planteamientos de los mismos.

Con respecto a los discursos expresados por el grupo de estudiantes participantes, se puede identificar que estos reconocen que la dinámica y problemáticas del mundo actual está demandando nuevas formas de intervención social desde Trabajo Social; desde discursos como la autogestión, la diversidad cultural, la responsabilidad social, la ética profesional e interdisciplinariedad. Sin embargo, se mantiene la tendencia de que estos son discursos expresados por docentes en el aula, quienes asumen de manera acrítica y como verdad absoluta por parte de estudiantes. Es decir, los discursos que afirman reconocer e impulsar los/as estudiantes de Trabajo Social en las entrevistas, no son reflexivizados sino repetidos. Aquellos y aquellas que han logrado hacer un proceso más crítico-reflexivo 
respecto a los discursos escuchados de sus profesores, ha sido porque se han vinculado a procesos investigativos y alternos a su formación en Trabajo Social, tales como semilleros y proyectos de investigación que así lo demandan.

$\mathrm{Si}$ bien existe una relación entre los discursos y prácticas de intervención social de los estudiantes de Trabajo Social, esta relación no trasciende la reproducción lingüística de principios y postulados, pues no se evidencia en estos, la forma como son apropiados y asumidos en su ejercicio académico aspectos tales como: equidad de género, derechos humanos, familia, política y responsabilidad social, etc.

A partir de estos hallazgos, se plantean algunos lineamientos para la formación profesional en Trabajo Social, que enfatizan en la intervención social.

\section{Lineamientos para una propuesta formativa en Trabajo Social con énfasis en la Intervención Social}

Para plantear algunos lineamientos en la formación profesional en Trabajo Social es preciso hacer énfasis en los procesos pedagógicos y formativos que se gestan en el ámbito universitario, la forma en que son asumidos y llevados a cabo por docentes y estudiantes y la manera como estos específicamente permean las representaciones sociales, discursos y prácticas frente a la intervención social. Considerando los hallazgos de la investigación, los/as estudiantes presentan debilidades con relación a que desconocen fundamentos teórico-conceptuales y autores respecto a la intervención social, así como una clara instrumentalización de los discursos y prácticas en torno a esta, por lo cual es preciso proponer directrices formativas que se orienten a superar dichas situaciones.

Por ello, es pertinente enfatizar en esta formación en los diferentes ejes de la misma, como es el caso de la intervención social, considerando elementos teóricos/conceptuales, metodológicos, éticos y contextuales. De esta manera se propone:

- Partir de la identificación, socialización y debate en torno a aspectos teóricos/conceptuales, metodológicos, éticos y contextuales acerca de la intervención social por parte de docentes y estudiantes.

- Generar procesos pedagógicos que se orienten a potenciar representaciones sociales, construcciones discursivas y prácticas de intervención social a partir 
de la indagación permanente por parte de docentes y estudiantes acerca de la forma como los asumen ellos y por las comunidades.

- Proponer la identificación y construcción de formas de intervención social más complejas, con los estudiantes y actores participantes en las prácticas académicas, a partir del impulso a procesos formativos que basados en estas investigaciones, exploren sus percepciones, discursos y prácticas de intervención social, a fin de que éstos sean cada vez más coherentes y efectivos. Lo anterior apunta en la formación y práctica académica de los estudiantes de Trabajo Social a construir y consolidar espacios y escenarios como semilleros y proyectos de investigación, prácticas de año social, grupos de debate, socialización de experiencias, que posibiliten el análisis y proposiciones frente a la intervención social desde sus diferentes ámbitos de trabajo y saberes.

Cabe señalar que, para el caso del programa de Trabajo Social de la Universidad de Cartagena, estos espacios y escenarios ya existen. De lo que se trata es de obtener el mayor aprovechamiento de los mismos. Según Beltrán (2010), debe propiciarse "Un encuentro permanente de reflexiones y experiencias, espacios de escritura, de debate y de formación desde los cuales construir colectivamente la idea de un Trabajo Social que responda a los contextos, sociales, económicos, políticos, culturales contemporáneos" (p.119).

Es preciso reiterar, que no todos los estudiantes participan en los distintos espacios de formación que se dan desde la universidad, de allí que se propone:

- Formar como pares académicos a estudiantes participantes de semilleros, proyectos y grupos de investigación para el intercambio de conocimientos y la socialización de experiencias con el resto de estudiantes no participantes en éstos, a fin de impulsar procesos inclusivos y equitativos, a propósito del análisis en torno a la intervención social.

- Estimular la producción escritural de estudiantes y docentes en torno a la intervención social, para proyectarla al escenario formativo en Trabajo Social a nivel local, regional, nacional e internacional.

La construcción y consolidación de escenarios de análisis, producción escrita y debates en la universidad, planeados con 
y desde los estudiantes, se convierte en una plataforma para proponer formas más complejas de intervención en Trabajo Social y su incidencia sobre las condiciones sociales, la organización y participación social.

Para finalizar, resta señalar la importancia de articular las percepciones, discursos y prácticas emergentes de los debates de Trabajo Social y reflexiones en torno a la intervención social, desde estudiantes y actores participantes, tanto en la formación profesional como en la intervención del Trabajo Social, hacia la construcción de formas de abordaje más comprensivas de la intervención social con el contexto inmediato, a partir del reconocimiento y respeto por cada uno de sus participantes. 


\section{Referencias}

Acevedo, Mariana. (2003). El aprendizaje ciudadano: desafío ineludible en la consolidación del sistema democrático. En Nora Aquin. (Comp.), Ensayos sobre Ciudadanía, En torno a la ciudadanía (pp157-165). Buenos Aires: Espacio Editorial.

Barranco, Carmen. (2004). La intervención en Trabajo Social desde la calidad integrada. Cuaderno de Trabajo Social, 12, 79102. Universidad de la Laguna. Recuperado de http: dialnet. unirioja.es

Beltrán, John. (2010). Trabajo Social y educación: reflexiones en torno al debate formativo y profesional contemporáneo. Biblioteca Digital Repositorio-Universidad Nacional de Colombia. Bogotá. Recuperado de http://www.bdigital.unal.edu.co/2638/

Botero, Patricia. (2008). Representaciones y Ciencias Sociales. Una perspectiva epistemológica y metodología. Buenos Aires: Espacio Editorial.

Carballeda, Alfredo. (2002). La Intervención en lo Social: exclusión e Integración en los nuevos escenarios sociales. Buenos Aires: Paidós.

Carballeda, Alfredo. (2007). Escuchar las prácticas: la supervisión como proceso de análisis de la intervención en lo social. Buenos Aires: Espacio Editorial.

Cartagena Cómo Vamos. (2010). Encuesta de percepción ciudadana sobre la calidad de vida en Cartagena. Recuperado de http://www.cartagenacomovamos.org/temp_downloads/ Presentacion\%202010\%20CCV\%20-\%20corregida.pdf.

Castañeda, Gloria. (2004). Enfoques de intervención social. Documento de trabajo. Recuperado de http: www.animacion juvenil.org.

Corvivienda. (2007). Plan maestro de vivienda. Cartagena: Alcaldía Mayor de Cartagena.

Londoño, Oscar. (2007). El análisis crítico del discurso: Una perspectiva crítica de lectura en estudiantes universitarios. Revista Electrónica Letralia, 1-12. Recuperado de http:// www.letralia.com/170/ensayo02.htm.

Martínez, Miguel. (2006). Hermenéutica y Análisis del Discurso como Método de Investigación Social. Recuperado de http://investigacionsocial-alquelquis.es.tl/Hermeneuticay-an\%E1lisis-del-Discurso-como-M-e2-todo-deInvestigaci\%F3n-Social.htm.

Pérez, Liliana. (2008). Autonomía, ética e intervención social. Una propuesta desde la ética del discurso y la acción comunicativa para el Trabajo Social contemporáneo. Revista de Trabajo Social, 10, 57-81. 
Reguillo, Rossana. (2004). El uso dialógico de las tecnologías en las sociedades dialógicas: una propuesta de democratización de los medios. Revista Nómadas, 2, 40-51.

Secretaría de Planeación Distrital. (2009). Cálculos Unidad plan de desarrollo. Base Sisben. Cartagena. Cartagena: Alcaldía Mayor de Cartagena.

Silva, Omer. (2002). El análisis del discurso según Van Dijk y los estudios de la comunicación. Universidad de la frontera, Temuco, Chile. Recuperado de http: //www.razonypalabra. org.mx/anteriores/n26/osilva.html.

Tejos, Braulio. (2005). Una mirada al concepto: intervención Social, en el marco de construcción del proyecto educativo de gesta. Documento de trabajo. Recuperado de http://docs.google. com/viewer?a=v\&q=cache:xDjEiheS.

Van Dijk, Teun. (2001). Discurso y Racismo: persona y sociedad. Universidad Alberto Hurtado. Instituto Latinoamericano de Doctrina y Estudios Sociales ILADES. Santiago de Chile. Recuperado de http://www.discursos.org/oldarticles/ Discurso\%20y\%20racismo.pdf. 


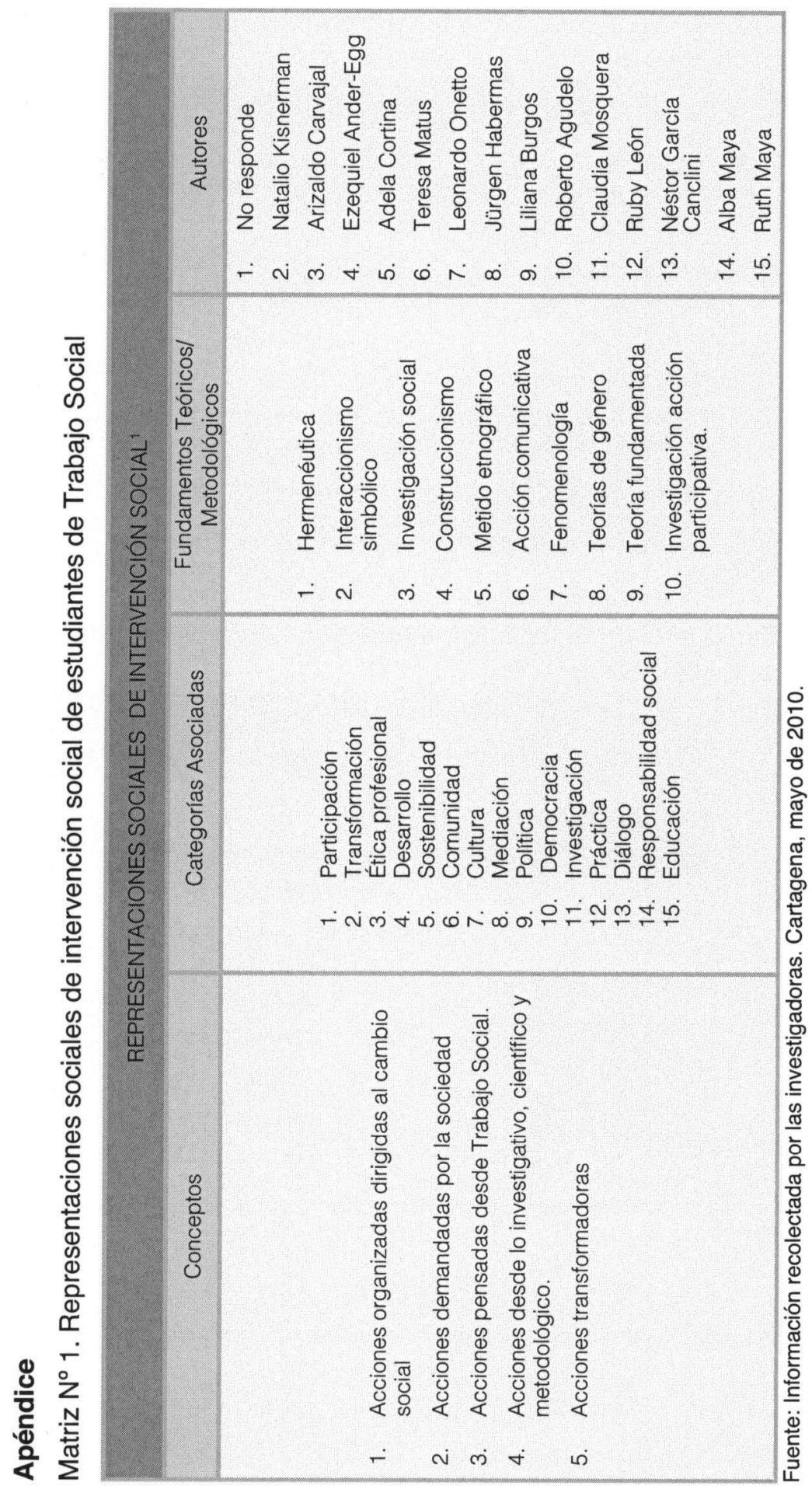


Matriz $\mathrm{N}^{\circ}$ 2. Discursos de intervención social de estudiantes de Trabajo Social

\begin{tabular}{|c|c|}
\hline \multicolumn{2}{|c|}{ DISCURSOS DE INTERVENCIÓN SOCIAL } \\
\hline $\begin{array}{l}\text { Discursos Contemporáneos } \\
\text { Reconocidos por Estudiantes }\end{array}$ & $\begin{array}{c}\text { Discursos Impulsados por } \\
\text { Estudiantes }\end{array}$ \\
\hline $\begin{array}{l}\text { 1. La ética profesional } \\
\text { 2. Autogestión de las } \\
\text { comunidades } \\
\text { 3. Responsabilidad social } \\
\text { 4. Restitución de derechos a } \\
\text { poblaciones vulnerables } \\
\text { 5. Interdisciplinariedad }\end{array}$ & $\begin{array}{l}\text { 1. Superación de la pobreza } \\
\text { 2. Equidad de género } \\
\text { 3. Reconocimiento derechos } \\
\text { 4. Diversidad cultural } \\
\text { 5. Autogestión } \\
\text { 6. Conciencia crítica }\end{array}$ \\
\hline
\end{tabular}

Fuente: Información recolectada por las investigadoras. Cartagena, mayo de 2010.

Matriz $N^{\circ}$ 3. Prácticas de intervención social de estudiantes de Trabajo Social.

\begin{tabular}{|c|c|}
\hline \multicolumn{2}{|c|}{ PRACTICAS DE INTERVENCIÓN SOCIAL } \\
\hline Prácticas Asociadas & Prácticas Impulsadas \\
\hline $\begin{array}{l}\text { 1. Equidad de género } \\
\text { 2. Derechos humanos } \\
\text { 3. Familia } \\
\text { 4. Lo político } \\
\text { 5. Responsabilidad social } \\
\text { 6. Población vulnerable } \\
\text { 7. Medio ambiente } \\
\text { 8. Desarrollo comunitario } \\
\text { 9. Gestión }\end{array}$ & $\begin{array}{l}\text { 1. Diversidad cultural } \\
\text { 2. Formación de líderes } \\
\text { 3. Equidad de género } \\
\text { 4. Fortalecimiento de la } \\
\text { autogestión } \\
\text { 5. Desarrollo social } \\
\text { 6. Pobreza } \\
\text { 7. Población vulnerable }\end{array}$ \\
\hline
\end{tabular}

Fuente: Información recolectada por las investigadoras. Cartagena, mayo de 2010.

Nota: la numeración obedece a la frecuencia con que fueron señaladas las respuestas por parte de los estudiantes entrevistados. 